\title{
Global burden of oesophageal and gastric cancer by histology and subsite in 2018
}

\author{
Melina Arnold (D) ,' Jacques Ferlay, ${ }^{1}$ Mark I van Berge Henegouwen (D) ,' \\ Isabelle Soerjomataram ${ }^{1}$
}

Additional material is published online only. To view please visit the journal online (http://dx.doi.org/10.1136/ gutjnl-2020-321600).

${ }^{1}$ Section of Cancer Surveillance, International Agency for Research on Cancer, Lyon, France

${ }^{2}$ Department of Surgery, Amsterdam UMC, University of Amsterdam, Cancer Center Amsterdam, Amsterdam, NoordHolland, The Netherlands

Correspondence to Dr Melina Arnold, Section of Cancer Surveillance, International Agency for Research on Cancer, Lyon 69008, France; arnoldm@iarc.fr

Received 27 April 2020 Revised 25 May 2020 Accepted 25 May 2020 Published Online First 30 June 2020

\begin{abstract}
Objectives To provide updated estimates of the global burden of oesophageal and gastric cancer by subsite and type.

Methods Using data from population-based cancer registries, proportions of oesophageal adenocarcinoma (OAC) and squamous cell carcinoma (OSCC) out of all oesophageal as well as cardia gastric cancer (CGC) and non-CGC (NCGC) out of all gastric cancer cases were computed by country, sex and age group. Proportions were subsequently applied to the estimated numbers of oesophageal and gastric cancer cases from GLOBOCAN 2018. Age-standardised incidence rates (ASR) were calculated.
\end{abstract}

Results In 2018, there were an estimated 572000 new cases of oesophageal cancer worldwide, 85000 OACs (ASR 0.9 per 100 000, both sexes combined) and 482000 OSCCS (ASR 5.3). Out of 1.03 million gastric cancers, there were an estimated 181000 cases of CGC (ASR 2.0) and 853000 cases of NCGC (ASR 9.2). While the highest incidence rates of OSCC, CGC and NCGC were observed in Eastern Asia (ASRs 11.1, 4.4 and 17.9, respectively), rates of $O A C$ were highest in Northern Europe (ASR 3.5). While globally OSCC and NCGC remain the most common types of oesophageal and gastric cancer, respectively, rates of OAC exceed those of OSCC in an increasing number of high-income countries.

Conclusions These updated estimates of the global burden of oesophageal and gastric cancer by subtype and site suggest an ongoing transition in epidemiological patterns. This work will serve as a cornerstone for policymaking and will aid in developing appropriate cancer control strategies.

\section{INTRODUCTION}

With more than 1.5 million new cases estimated in 2018, gastric cancer (GC) and oesophageal cancer belong to the most commonly diagnosed malignancies worldwide. ${ }^{12}$ Both are associated with a high disease-related mortality, resulting in close to 1.3 million deaths in the same year. ${ }^{1-3}$ Given their anatomic proximity, both sites share a number of risk factors and epidemiological features, but also display distinct geographical and temporal patterns in incidence. Differences in the distribution of tumour subtypes across populations have been found to partly explain these differentials in the burden from GC and oesophageal cancer. ${ }^{45}$

\section{Significance of this study}

What is already known about this subject?

- Trends and epidemiological patterns in the incidence of gastric and oesophageal cancer differ greatly by subtype: steep incidence increases in oesophageal adenocarcinoma (OAC) in highly developed countries are contrasted with incidence declines in oesophageal squamous cell carcinoma (OSCC) in many parts of the world; incidence of cardia gastric (CGC) and non-cardia (NCGC) cancers following unclear patterns and largely declining, respectively.

- Previous global estimates for 2012 showed that OSCC and NCGC are the predominant types of oesophageal and gastric cancer, with OAC representing an important proportion of oesophageal tumour in a number of highincome countries.

What are the new findings?

- Bringing together the most recent evidence from population-based cancer registries around the world and estimations of the global cancer burden in 2018, we estimated a total of 85000 , 482000,181000 and 853000 cases of OAC, OSCC, CGC and NCGC, respectively, worldwide.

- Country-level estimates reveal and confirm high-incidence area for each of the four sites and patterns across levels of human development.

- While providing up-to-date estimates, this study confirms previous findings of OSCC and NCGC remaining the most common types of oesophageal and gastric cancer to date.

How might it impact on clinical practice in the foreseeable future?

- This quantification of the global burden of subtype-specific/subsite-specific incidence provides an important evidence base for clinicians and health policy-makers to plan appropriate cancer control now and to impact cancer burden in the future.

- As incidence patterns shift, driven by ongoing changes in the prevalence of underlying risk factors, changing patient profiles will require an adaptation of current management and resource allocation.
To cite: Arnold M, Ferlay J van Berge Henegouwen $\mathrm{Ml}$ et al. Gut

2020;69:1564-1571. 
Typically, two main histological subtypes of oesophageal cancer (adenocarcinoma (OAC) and squamous cell carcinoma (OSCC)) and two subsites of GC (cardia (CGC) or proximal GC and non-cardia (NCGC) or distal GC) are distinguished. While OSCC occurs mostly in stratified squamous epithelium lining the upper two-thirds of the oesophagus, OAC typically develops in the lower third of the oesophagus and originates predominantly from Barrett mucosa. ${ }^{6}$ Whereas CGC cancers arise in the proximal stomach, close to the oesophageal-gastric junction, NCGC cancers occur in more distal regions of the stomach. All four subtypes/subsites have both distinct but also shared aetiological pathways. While smoking and heavy alcohol consumption have been linked to OSCC, CGC and NCGC, the rising incidence rates of $\mathrm{OAC}$ in high-income countries have been mainly attributed to increasing body weight and GORD. ${ }^{78}$ Obesity has also been associated with CGC, whereas according to recent estimates about $90 \%$ of all NCGC are due to infection with Helicobacter pylori. ${ }^{9}$ Given these differences but also similarities in epidemiological characteristics, there is increasing interest in describing the worldwide burden of oesophageal and GC subsites separately.

Building on our previous estimation of the burden from oesophageal and stomach (gastric) cancer by subtype/subsite, ${ }^{45}$ we here present a comprehensive, updated assessment of the burden, incorporating data from population-based cancer registries and estimates of the global cancer burden from GLOBOCAN 2018, by country, region and Human Development Index (HDI).

\section{METHODS}

The estimated total numbers of new oesophageal cancer and GC in 185 countries in 2018 by age, sex and country were obtained from the GLOBOCAN 2018 database. ${ }^{10}$ To obtain site-specific proportions, we used data from the Cancer Incidence in Five Continents Vol. XI database, ${ }^{11}$ which contains cancer incidence data from 2008 to 2012 from 343 cancer registries worldwide, complemented with data submitted by population-based cancer registries for the estimation of the GLOBOCAN 2018 database, and supplemented by updated data from the African Cancer Registry Network for the Sub-Saharan African region. In total, incidence data from 95 countries were available. For oesophageal cancer, the two main histological types were defined according to the third edition of the International Classification of Diseases for Oncology (ICD-O-3): OSCC: 8050-8078, 8083-8084; OAC: $8140-8141,8143-8145,8190-8231,8260-8265,8310$, $8401,8480-8490,8550-8552,8570-8574,8576$. For GC, subsites were defined according to the ICD, 10th Revision (ICD10) as follows: CGC (C16.0) and NCGC (C16.1-9), whereby cancers with overlapping or undefined topography were considered NCGC. Populations with more than $75 \%$ of all GC cases coded as 'not otherwise specified' (C16.9, herein referred to as 'NOS') were excluded.

In a first step, sex-specific and age-specific $(<65$ and $\geq 65$ years) proportions of all four subtypes (OAC, OSCC, CGC and NCGC) were computed for countries with at least two cases in each stratum of sex and age, based on cases diagnosed with microscopic verification. Age strata were chosen to reflect age-specific differences in the distribution of subtypes. Where data were available from multiple cancer registries within one country, these were aggregated to obtain estimated national proportions. The total of all carcinomas (for oesophageal cancer: OAC + OSCC + other carcinomas; for GC: CGC + NCGC) was used as the denominator. For oesophageal cancer, national-level proportions were computed for 60 countries and for GC for 49 countries. A full list of methods used for individual countries is available in online supplementary table 1 .

For the remaining countries without sufficient populationbased registry data or where data were excluded due to small numbers ( $n=125$ for oesophageal cancer; $n=136$ for GC), regional proportions were calculated. This was done by aggregating country-level data from the first step. Countries were categorised into regions based on UN geographical areas: SubSaharan Africa, Northern Africa, Caribbean and Central America, South America, Northern America, Eastern Asia (including China), South-Eastern Asia, South-Central Asia (including India), Western Asia, Eastern Europe, Northern Europe, Western Europe, Southern Europe and Oceania.

The resulting proportions were subsequently multiplied with the total number of GC and oesophageal cancer cases by 5 -year age group, sex and country as presented in GLOBOCAN 2018. Age-standardised incidence rates (ASRs per 100000 person years) by sex were calculated for each country and region and according to the four categories of $\mathrm{HDI}^{12}$ in 2017 using the direct method and the World Standard Population. ${ }^{13}$

In secondary analyses, the impact of excluding GC cases with overlapping or unspecified topography (C16.8-9), as well as the exclusion of histological types other than OAC and OSCC in the calculation of the subtype-specific proportions, was explored and results of the overall burden compared.

\section{Patient and public involvement}

As this work is a retrospective study involving examination of secondary cancer data only, patients were not involved in the design and conduct of this research.

\section{RESULTS}

\section{Oesophageal cancer}

In 2018, there were an estimated 572000 new cases of oesophageal cancer worldwide, composed of 85000 (15\%) cases of OAC, corresponding to an ASR of 0.9 per 100000 and 482000 (84\%) OSCC cases with an ASR of 5.3 per 100000 . About 70\% of all oesophageal cancers occurred in men, who had proportionally more OACs than women (16\% vs $11 \%)$. OSCC represented the most common subtype of oesophageal cancer in all world regions, except for Northern America, Northern Europe and Oceania, where the number (and proportion) of OAC cases exceeded those of OSCC (table 1, online supplementary table 2 and figure 1).

For both sexes combined, the majority of all OAC cases occurred in Eastern Asia (29000 cases), followed by Northern America (15 000 cases) and Western Europe (8000 cases). Yet, the highest incidence rates of OAC were observed in Northern Europe (ASR 3.5) and Northern America (2.2; figure 2). On the country level, China (27000 cases) and the USA (13000 cases) contributed most to this burden (online supplementary table 3), mostly because of their big populations but also because of high incidence rates. The highest incidence rates of OAC were observed in the Netherlands and the UK (ASR 4.4 per 100 000) and OAC rates exceeded OSCC rates in 21 countries (figures 3 and 4 and). With more than $45 \%$ of all cases occurring in very high HDI countries, the rates of OAC followed a clear gradient across HDI categories, with the lowest rates being observed in low and medium HDI countries (online supplementary figures 1-3).

For OSCC, the overall burden was concentrated in Asia, where more than $80 \%$ of the global cases occurred in 2018 (403 000 cases; table 1 and figure 1). Over 277000 cases of 


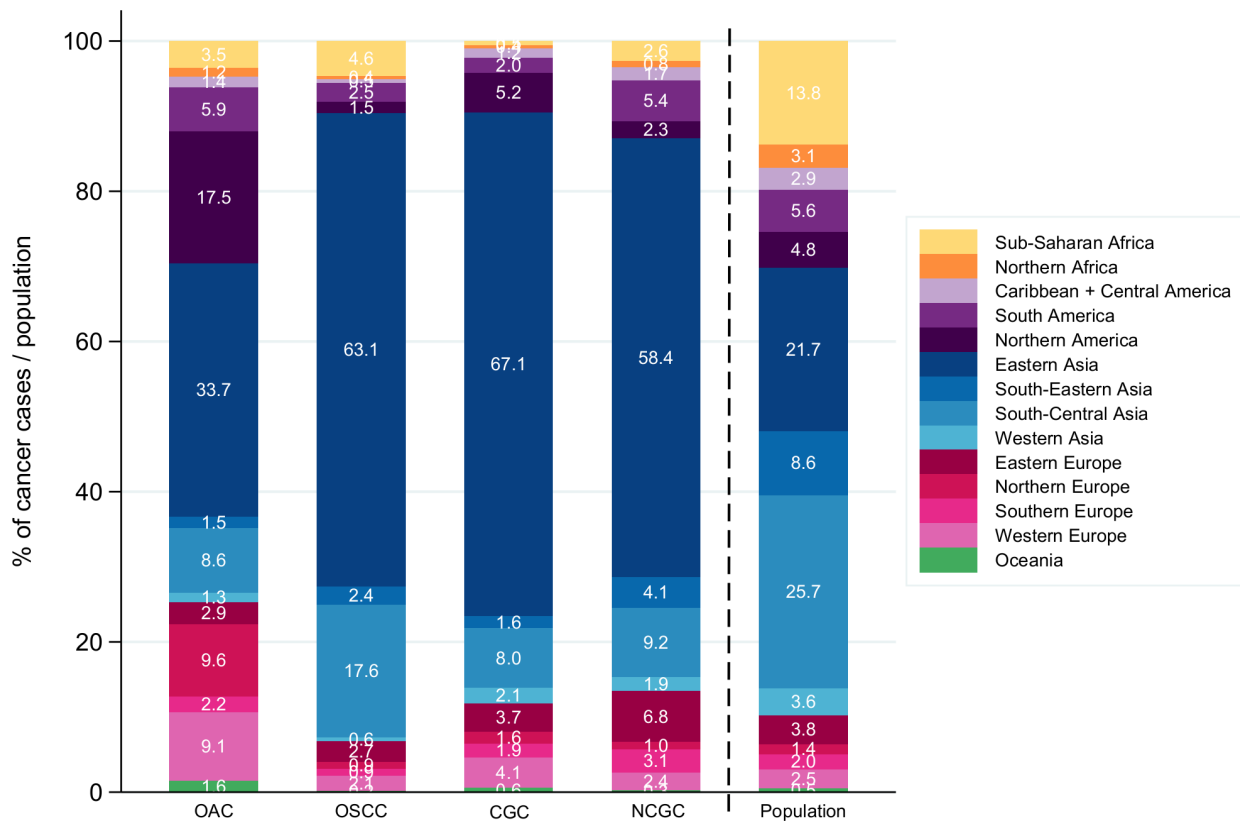

Figure 1 Global estimates of oesophageal and gastric cancer cases for each subsite/subtype by world region for both sexes combined, 2018. CGC, cardia gastric cancer; NCGC, non-cardia gastric cancer; OAC, oesophageal adenocarcinoma; OSCC, oesophageal squamous cell carcinoma.

OSCC were estimated for China alone (online supplementary table 3). Furthermore, Sub-Saharan Africa carried a high burden from OSCC, with over 22000 cases in 2018. Incidence rates ranged from 11.1 per 100000 in Eastern Africa (ASR > 16 in Mongolia, Malawi and Kenya) to 1.0 per 100000 in Northern Africa, Northern America and the Caribbean and Central America (figures 2 and 3). No clear patterns were observed when analysing the burden from OSCC by HDI. While countries with a very high HDI carried proportionally less cases, incidence rates were highest in high HDI countries (ASR 9.1) and did not differ much across the remaining HDI categories (online supplementary figures 1-3).

\section{Gastric cancer}

Out of 1.03 million GCs in 2018, there were an estimated 181000 (18\%) cases of CGC (ASR 2.0) and 853000 (82\%) cases of NCGC (ASR 9.2). While two-thirds of all GCs worldwide occurred in men, this proportion was slightly higher for CGC where an estimated three quarters of all cases were among men. NCGC was the
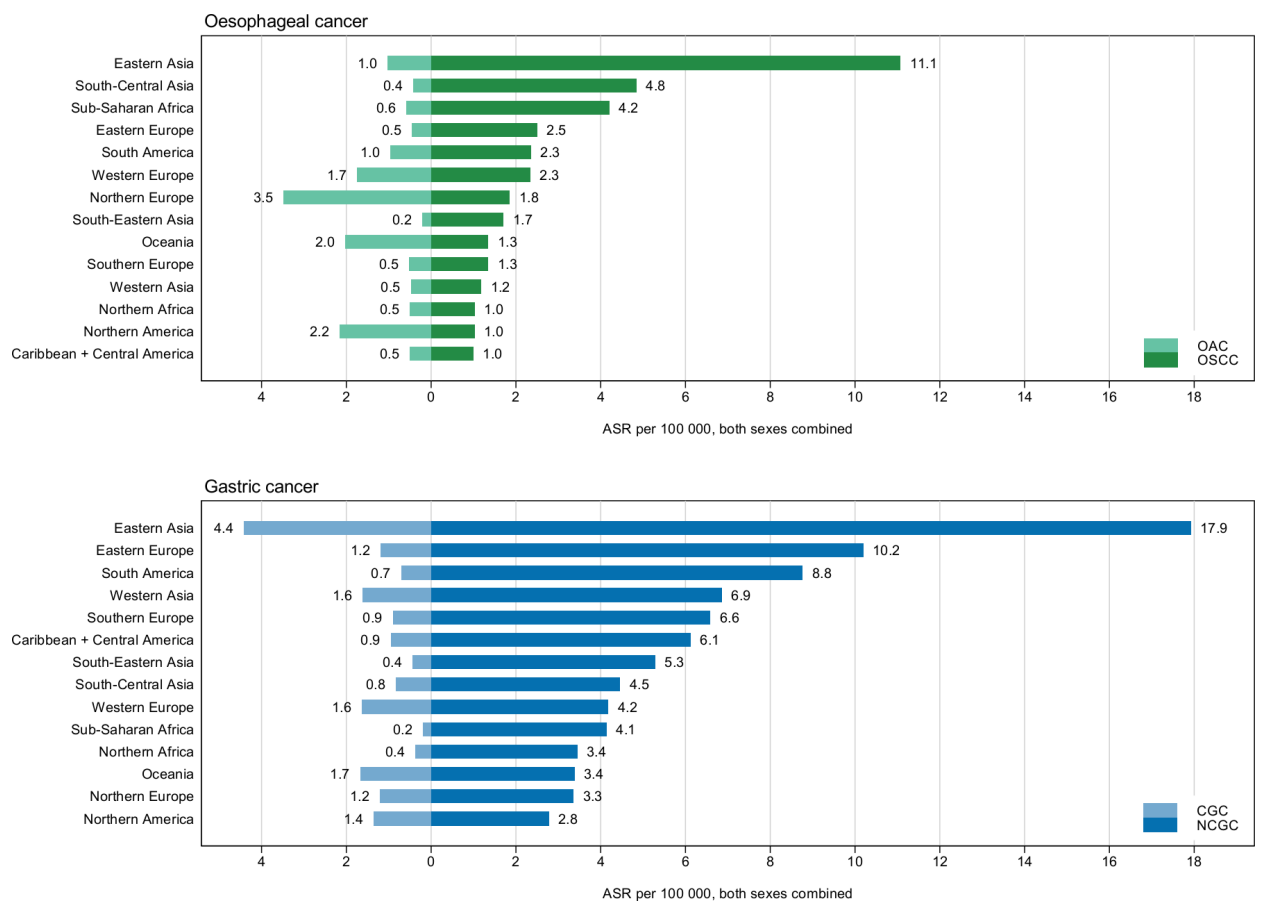

Figure 2 Age-standardised incidence rates (ASR, World) per 100000 of oesophageal and gastric cancer for each subsite/subtype by world region for both sexes combined, 2018. CGC, cardia gastric cancer; NCGC, non-cardia gastric cancer; OAC, oesophageal adenocarcinoma; OSCC, oesophageal squamous cell carcinoma. 

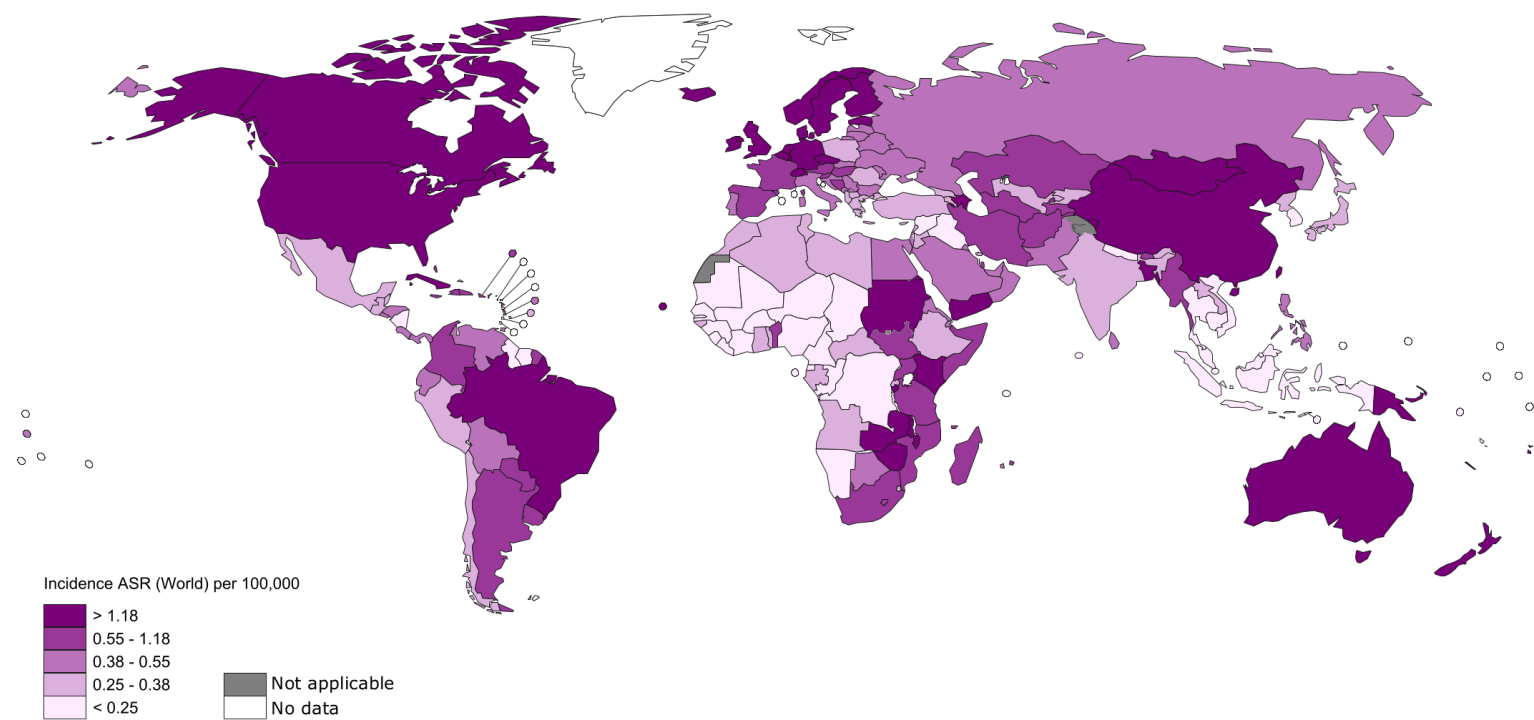

All rights reserved. The designations employed and the presentation of the material in this publication do not imply the expression of any opinion whatsoever on the part of the World Health Organization / International Agency for Research on Cancer concerning the legal status of any country, territory, city or area or of its authorities, or concerning the delimitation of its frontiers or
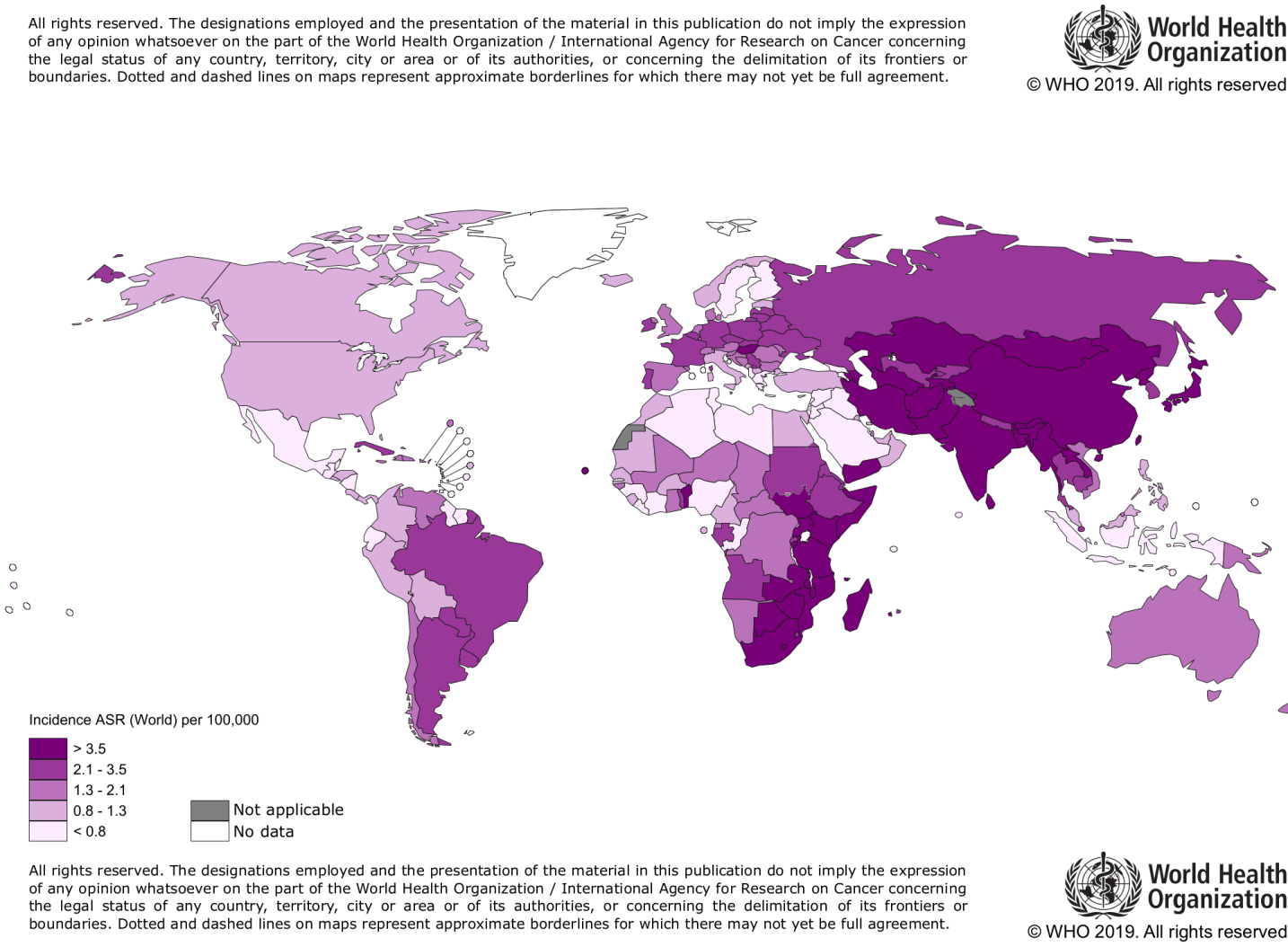

Figure 3 Age-standardised incidence rates (ASR, World) per 100000 of adenocarcinoma (upper map) and squamous cell carcinoma (lower map) of the oesophagus, both sexes combined, 2018.

most common subtype of GC globally (853000 cases, $82 \%)$ and across all world regions, representing between $68 \%$ and $96 \%$ of all GC cases (table 1, online supplementary table 2 and figure 1).

For both CGC and NCGC, the vast majority of cases occurred in Asia, together representing over $70 \%$ of the global case burden of both subsites (figure 1). In the case of CGC, about 121000 cases (67\%) occurred in Eastern Asia (whereof 110000 in China), followed by South Central Asia (14000 cases, 8\%) and Northern America (9000 cases, 5\%). Incidence rates of CGC were highest in Eastern Asia (4.4 per 100 000), followed by Oceania, Western Europe and Western Asia (ASR 1.7, 1.6 and 1.6, respectively) and lowest in Sub-Saharan Africa (ASR 0.2; figure 2). On the country level, the risk of CGC was highest in China and Iran (ASR 5.0; figure 5, online supplementary table 4). Most CGC cases occurred in high HDI countries (comprising China), which was also reflected in incidence rates that were highest in this group of countries (ASR 3.7; online supplementary figures 1 and 2) and a significant trend of increasing rates with increasing HDI (online supplementary figure 3).

For NCGC on the other hand, close to 500000 cases occurred in Eastern Asia alone (58\% of the global NCGC burden) and another 130000 in the remaining Asian regions. This was followed by Europe (113000 cases, 13\%), South America, the Caribbean and Central America (61000 cases, 7\%; table 1 and figure 1). Incidence rates ranged from 17.0 per 100000 in Eastern Asia (ASR $>25$ in Korea, Mongolia and Japan) to less than 3 per 100000 


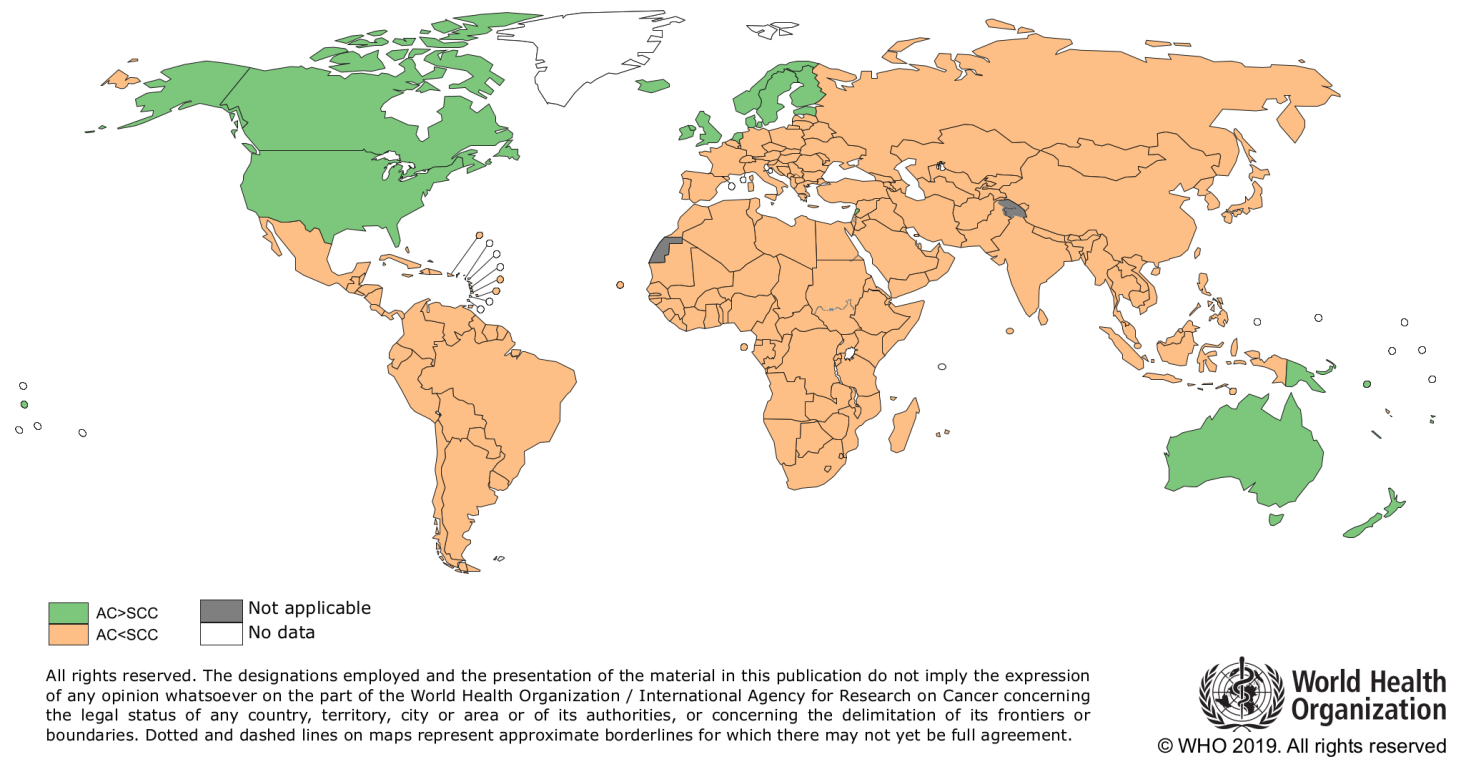

Figure 4 Highlighting countries where age-standardised incidence rates (ASR, World) per 100000 person years of oesophageal adenocarcinoma (AC) exceed those of squamous cell carcinoma (SCC), both sexes combined, 2018.

in Northern America (online supplementary table 4, figures 2 and 5). More than $70 \%$ of all NCGC cases occurred in high and very high HDI countries, with incidence rates being highest in these two HDI groups (ASR 13.0 and 9.1, respectively; online supplementary figures 1-3).

\section{Secondary analyses}

Excluding histologies other than specified adenocarcinomas and squamous cell carcinomas (representing 1\% of all oesophageal cancer cases), when estimating from the global burden from OAC (86000 cases; $15 \%)$ and OSCC (486000 cases; $85 \%)$, only marginally affected overall results (online supplementary tables S5-7). However, excluding GCs at overlapping or unspecified subsites when computing site-specific proportions gave a somewhat different picture for the global burden of CGC (316000 cases; 31\%) and NCGC (717000 cases; 69\%), particularly in Sub-Saharan Africa, South America and South Central Asia where tumours with unspecified topography represented a considerable proportion of all GCs (online supplementary tables 5-8).

The distribution of subtypes across world regions and general patterns in incidence rates was similar in men and women (online supplementary figures 4-12).

Table 1 Estimated numbers and proportions of oesophageal and gastric cancers for both sexes combined in 2018 by subtype, world region and Human Development Index (HDI)

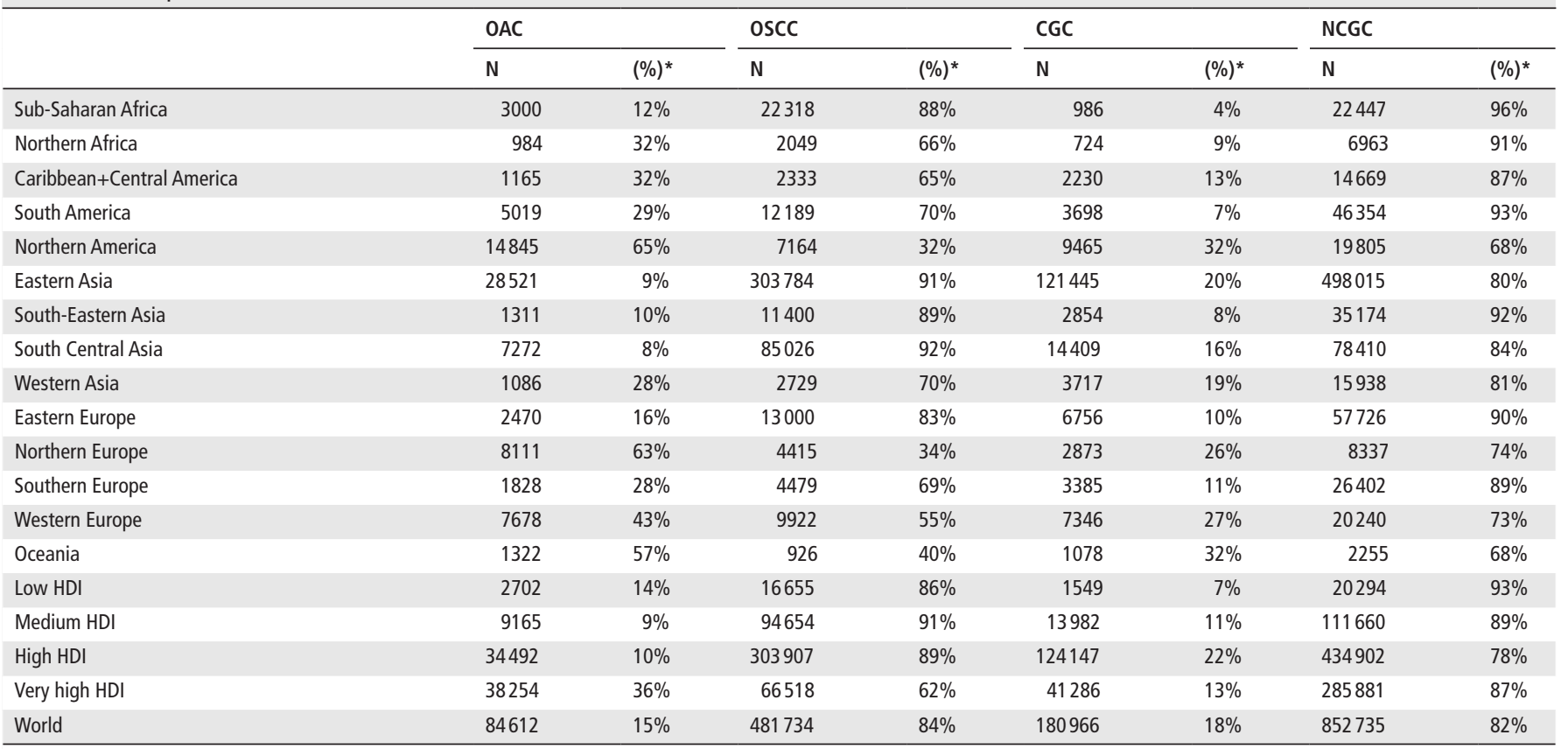

*Row percentage out of all oesophageal cancers (International Classification of Diseases (ICD)-10 C15) for oesophageal adenocarcinoma (OAC) and oesophageal squamous cell carcinoma (OSCC) and out of all gastric cancers (ICD-10 C16) for cardia gastric cancer (CGC) and non-cardia gastric cancer (NCGC); other oesophageal subtypes are not shown. 

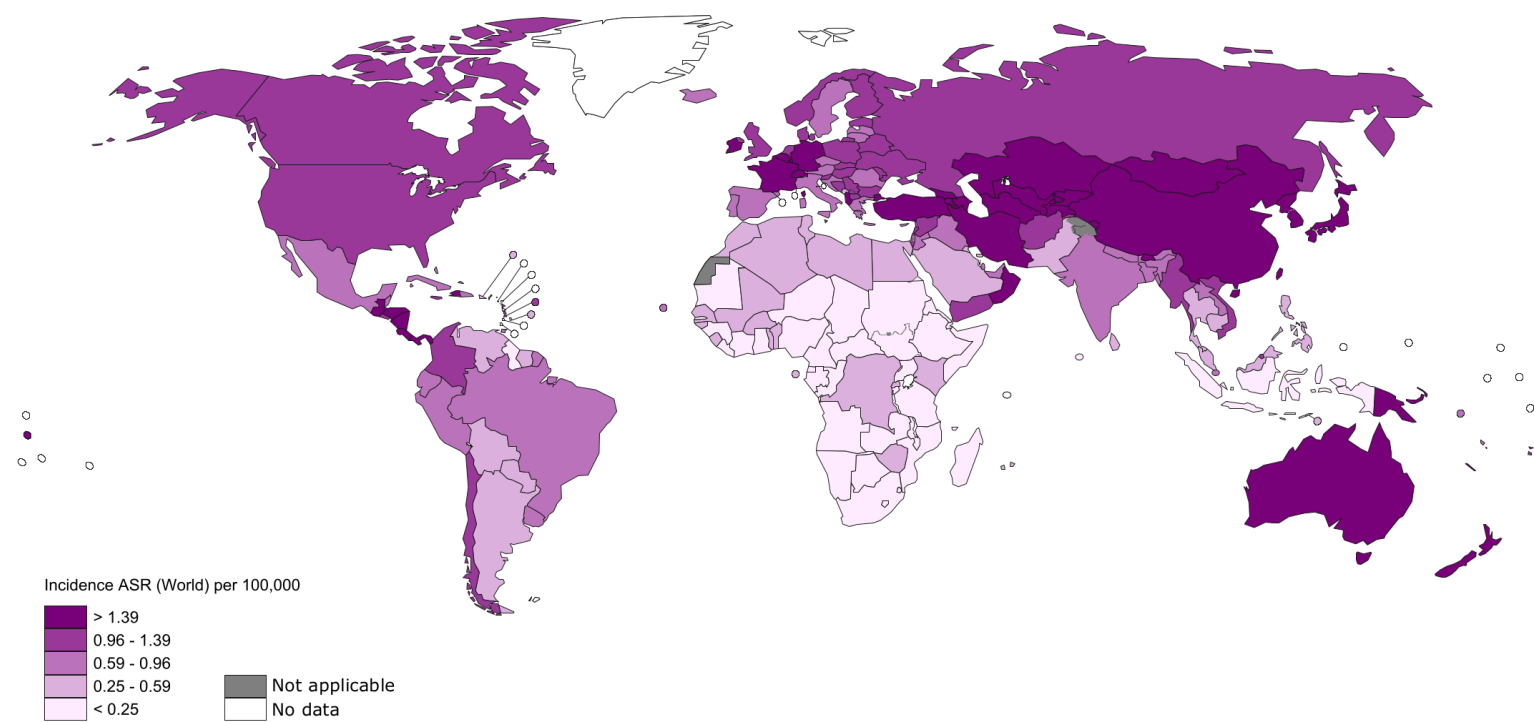

All rights reserved. The designations employed and the presentation of the material in this publication do not imply the expression of any opinion whatsoever on the part of the World Health Organization / International Agency for Research on Cancer concerning the legal status of any country, territory, city or area or of its authorities, or concerning the delimitation of its frontiers
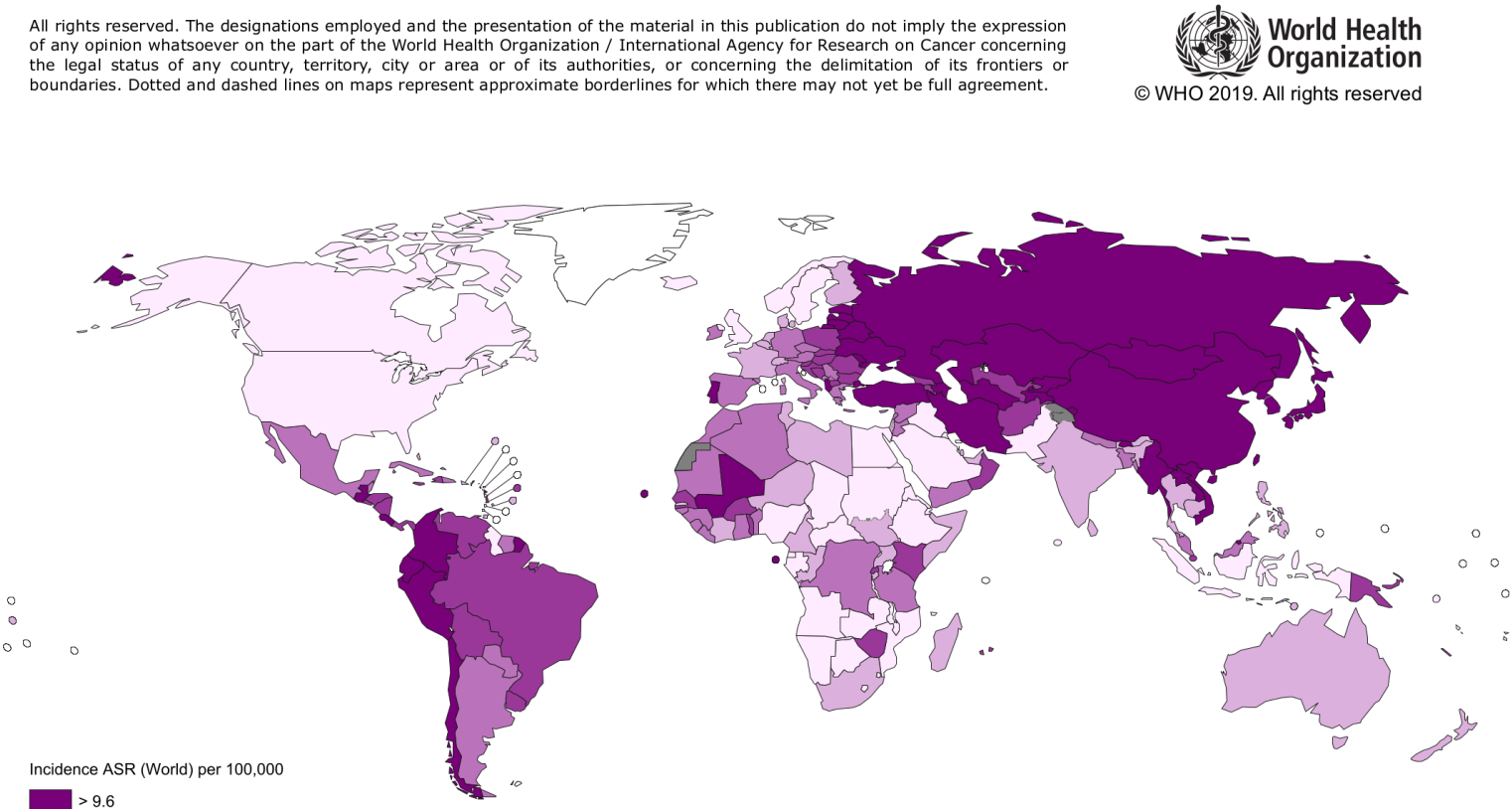

$>9.6$

$4.2-6.3$

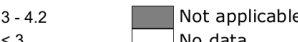

All rights reserved. The designations employed and the presentation of the material in this publication do not imply the expression of any opinion whatsoever on the part of the World Health Organization / International Agency for Research on Cancer concerning the legal status of any country, territory, city or area or of its authorities, or concerning the delimitation of its frontiers or
boundaries. Dotted and dashed lines on maps represent approximate borderlines for which there may not yet be full agreement.

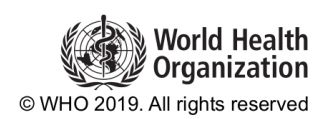

Figure 5 Age-standardised incidence rates (ASR, World) per 100000 of cardia (upper map) and non-cardia (lower map) gastric cancer, both sexes combined, 2018.

\section{DISCUSSION}

In this study presenting most up-to-date estimates of the global incidence of oesophageal cancer and GC by subtype and subsite, we provide a comprehensive picture of the current epidemiological profile of these two cancers. While OSCC remains the most common subtype of oesophageal cancer globally ( $84 \%$ of all cases), with highest incidence rates in Eastern Asia and Eastern Africa, the number of OAC cases exceeds those of OSCC in a substantial number of high-income countries and rates are highest in Northern Europe, North America and Oceania. For GC, NCGC continues to prevail globally (82\% of all cases), with a high case burden in Asia, but also in South and Central America. Patterns for CGC were slightly different from NCGC, with the highest burden in Eastern Asia and parts of Oceania and Western Asia.
Our results build on previous global assessments of the burden of these cancers ${ }^{45}$ and are in line with other studies that have evaluated trends and patterns in more confined, national settings. For OAC, high-incidence areas and increasing trends have previously been reported in high-income countries such as the USA, Australia and the UK. ${ }^{14-18}$ These have largely been attributed to increasing levels of (central) obesity that is known to be one of the main causes of GORD-which can lead to long-term complications, for example, Barrett's oesophagus, increasing the risk of OAC. At the same time, $H$. pylori infection-the main causal risk factor for $\mathrm{GC}$ - has been postulated to protect from OAC through the development of atrophic gastritis and associated reductions in the acidity and peptic activity of gastric juice as well as an attenuation of acid pockets in the gastro-oesophageal 
junction. ${ }^{7} 1920$ Continuing decreases in the prevalence of $H$. pylori may therefore contribute to increases the concentration of acid, pepsin and bile in gastric refluxate, the main factors damaging to the oesophageal mucosa, which are known to promote OAC development. It has previously been shown that particularly abdominal obesity may lead to an aggravation of continuous acid exposure linked to immunohistological changes of junctional squamous mucosa and supporting evidence that obesity acts as a risk factor for both OAC and CGC. ${ }^{21}$

According to recent projections, the incidence of OAC is set out to continue to increase and to become the predominant type of oesophageal cancer in a growing number of high-income and very high-income countries. ${ }^{18}$ Globally, however, OSCC remains the most common, particularly in high-incidence areas such as Northern Iran, ${ }^{22}$ Central Asia and China ${ }^{23}$ (together forming the so-called 'oesophageal cancer belt') as well as parts of Eastern Africa. ${ }^{24-26}$ While tobacco smoking and heavy alcohol consumption remain to be the two main risk factors for OSCC and have been found to be responsible for the majority of OSCC cases in high-income countries, ${ }^{27} 28$ other risk factors such as hot beverage drinking, opium use and indoor air pollution dominate in high-incidence areas such as Iran or countries in Eastern Africa such as Kenya. ${ }^{29}{ }^{30}$ To date, population-based screening using endoscopy has not been recommended given the associated relatively high cost and risk of complication, and non-endoscopic cell-sampling methods have been found to lack the necessary specificity. ${ }^{31}$ Clinical trials of other, less-invasive screening methods (eg, inflatable balloons and sponges and exhaled breath examination ${ }^{32}$ ) are currently underway, and may be a more favourable approach to screen for oesophageal cancer in the future, given improvements in specificity and risk stratification. ${ }^{313}$ For now, primary prevention measures, including obesity control in high-income settings and continuing interventions that seek to reduce the smoking and alcohol prevalence in the community, remain key to reducing the incidence of oesophageal cancer.

H. pylori infection remains the main causal risk factor for GC and has been attributed to $90 \%$ of all NCGC and $20 \%$ of all GC in 2018. ${ }^{9}$ Other risk factors of CGC, which typically comprise a mixture of tumours whose origin may lie in either the lower oesophagus or the proximal stomach, are GORD and obesity in western populations (resembling aetiological factors attributed to OAC). ${ }^{34}{ }^{35}$ These risk factor patterns are also reflected geographically, with CGC being most common in Central Asia, but high incidence rates also seen in Oceania and parts of Western Europe. While the incidence of GC has been falling since decades, mostly due to reductions in $H$. pylori prevalence, and has become rare in some world regions, its burden is set out to remain high in many high-income countries such as Korea. ${ }^{36}$ These trends contrasted the increasing incidence observed in younger generations in low-incidence countries such as the UK and the US, populations with typically a low prevalence of $H$. pylori infection. ${ }^{36-38}$ Potential reasons for this risk transition comprise changes in the microbiome associated with modern lifestyle, in particular increases in obesity, which has been linked with CGC risk. ${ }^{39}$ Although routine screening for GC in low-risk populations has not proven to be cost effective, the implementation of population-based screening in high-risk regions has shown some promise and nationwide GC screening programmes have been available in Korea and Japan over several decades. ${ }^{40-43}$ In parallel, the cost effectiveness of $H$. pylori eradication is being explored in several ongoing randomised controlled trials indicating that treatment lowers GC risk $^{44}$ and may aid in GC prevention in some settings. ${ }^{45}$
While this study represent the best of our knowledge on the global burden of oesophageal and stomach cancer by subtype and subsite, several limitations should be noted. First, the estimation of subtype-specific and subsite-specific data was limited by the extent to which histological and topographical information was available from each cancer registry. While oesophageal cancer data by histology were relatively complete, it represented a clear challenge for stomach cancer, where on average $49 \%$ of all cancers were categorised as overlapping or unspecified location (C16.8-9). We tried to mitigate this problem by including only registries where this proportion did not exceed $75 \%$ and by carefully scrutinising each data set in its regional and national context. In this updated assessment, we assigned C16.8-9 cases to tumours of the distal stomach, that is, NCGC, which differs from our previous approach and led to a slightly lower proportion of CGC (18\% vs $27 \%)$. $^{5}$ This decision was based on the assumption that cardia stomach cancers would be more likely to be recognised and coded as such, while it can be more difficult to assign an exact anatomical location to an often large tumour in the distal part of the stomach. Exact tumour location is typically derived from surgical reports-a procedure that is often not carried out for GCs as they are often diagnosed at late stage. By choosing this approach, we may have overestimated the burden of NCGC in some settings. In secondary analyses, we demonstrated the impact of excluding GCs with overlapping or unspecified location in the estimation of proportions by subsite, resulting in a much larger proportion of cardia cancers, which is likely an overestimation. We believe that the impact of the assumptions made in our main analysis is more realistic and offers an advantage over excluding GCs with unspecified topography.

Second, while the distinction between CGC and NCGC is certainly challenging, it can also be difficult to determine whether a tumour originated in the stomach or in the oesophagus. ${ }^{46} 47$ Depending on the exact location, both OAC and GC can together be considered cancers of the gastro-oesophageal junction, which are sometimes considered to represent the same clinical entity. ${ }^{47}$ It has been postulated that part of the increasing incidence of OAC might be due to more frequent classification of cancers at the gastro-oesophageal junction as oesophageal rather than GCs. This would however only account for a small proportion of cases and would not affect the overall patterns we see. Together these issues highlight the need for more accurate subsite-specific cancer data that are essential to solidify our knowledge on the cancer burden, especially in world regions where such data are sparse.

Finally, data obtained from GLOBOCAN represent estimates themselves, meaning that they should be interpreted with caution. ${ }^{10}$ The population-based data from cancer registries we used to obtain subsite and subtype proportions were limited to countries where such data were available. Where country-specific proportions were not available, regional information was aggregated to obtain national proxies (see also online supplementary table 5). Hence, heterogeneity across these countries may not be adequately represented.

By presenting incidence estimates of oesophageal and GC by subtype and subsite, this study provides a comprehensive, updated picture of the global burden of upper GI cancers, which is highly relevant for cancer control as well as for clinical practice. Our study showed that globally, OSCC and NCGC remain the most common types of oesophageal cancer and GC, respectively; yet, distinct epidemiological patterns can be observed. While OAC dominates in high-income countries where it has overtaken OSCC rates, OSCC, CGC and NCGC continue to show a strong predominance in Eastern Asia where rates are among the highest worldwide. These results reinforce the need for and the power of primary and secondary prevention measures, which together remain the most 
important tools to control both oesophageal and GCs now and in the future.

Twitter Mark I van Berge Henegouwen @mivanbh

Acknowledgements The authors gratefully acknowledge all cancer registries and their staff who have contributed in sharing their data needed for this study. They would like to thank the African Cancer Registry Network and all its collaborators for the provision of data.

Contributors Study concept and design: MA, JF and IS. Analysis and interpretation of data: all authors. Drafting the manuscript: MA and IS. Critical revision of the manuscript for important intellectual content: all authors.

Funding The authors have not declared a specific grant for this research from any funding agency in the public, commercial or not-for-profit sectors.

Disclaimer Where authors are identified as personnel of the International Agency for Research on Cancer/WHO, the authors alone are responsible for the views expressed in this article and they do not necessarily represent the decisions, policy or views of the International Agency for Research on Cancer/WHO.

Competing interests MlvBH reports acting as consultant for Medtronic, Johnson \& Johnson and Mylan as well as grants from Olympus en Stryker, outside the submitted work.

Patient and public involvement Patients and/or the public were not involved in the design, or conduct, or reporting, or dissemination plans of this research.

Patient consent for publication Not required.

Provenance and peer review Not commissioned; externally peer reviewed.

Data availability statement Data are available in a public, open access repository. Data are available on https://gco.iarc.fr/ and on https://ci5.iarc.fr/Default. aspx.

ORCID iDs

Melina Arnold http://orcid.org/0000-0003-1700-6831

Mark I van Berge Henegouwen http://orcid.org/0000-0001-8689-3134

\section{REFERENCES}

1 Ferlay J, Ervik M, Lam F, et al. Global cancer Observatory: cancer today. Lyon, France: International Agency for Research on Cancer, 2018. https://gco.iarc.fr/today

2 Bray F, Ferlay J, Soerjomataram I, et al. GLOBOCAN estimates of incidence and mortality worldwide for 36 cancers in 185 countries. CA Cancer I Clin 2018:2018:394-424

3 Arnold M, Abnet CC, Neale RE, et al. Global burden of 5 major types of gastrointestinal cancer. Gastroenterology 2020. doi:10.1053/j.gastro.2020.02.068. [Epub ahead of print: 02 Apr 2020].

4 Arnold M, Soerjomataram I, Ferlay J, et al. Global incidence of oesophageal cancer by histological subtype in 2012. Gut 2015;64:381-7.

5 Colquhoun A, Arnold M, Ferlay J, et al. Global patterns of cardia and non-cardia gastric cancer incidence in 2012. Gut 2015;64:1881-8.

6 Enzinger PC, Mayer RJ. Esophageal cancer. N Engl J Med 2003;349:2241-52.

$7 \mathrm{McColl} \mathrm{KEL}$. What is causing the rising incidence of esophageal adenocarcinoma in the West and will it also happen in the East? J Gastroenterol 2019;54:669-73.

8 Thrift AP. The epidemic of oesophageal carcinoma: where are we now? Cancer Epidemiol 2016;41:88-95.

9 de Martel C, Georges D, Bray F, et al. Global burden of cancer attributable to infections in 2018: a worldwide incidence analysis. Lancet Glob Health 2020;8:e180-90.

10 Ferlay J, Colombet M, Soerjomataram I, et al. Estimating the global cancer incidence and mortality in 2018: GLOBOCAN sources and methods. Int J Cancer 2019:144:1941-53.

11 Bray F, Colombet M, Mery L, et al. Cancer incidence in five continents, vol. XI (electronic version. Lyon: International Agency for Research on Cancer, 2017.

12 Human Development Indices and Indicators - 2018 Statistical Update. New York: United Nations Development Programme, 2018.

13 Segi M. Cancer mortality for selected sites in 24 countries (1950-57. Sendai, Japan: Department of Public Health, Tohoku University of Medicine, 1960.

14 Cook MB, Chow W-H, Devesa SS. Oesophageal cancer incidence in the United States by race, sex, and histologic type, 1977-2005. Br J Cancer 2009;101:855-9.

15 Offman J, Pesola F, Sasieni P. Trends and projections in adenocarcinoma and squamous cell carcinoma of the oesophagus in England from 1971 to 2037. Br J Cancer 2018:118:1391-8

16 Thrift AP, Whiteman DC. The incidence of esophageal adenocarcinoma continues to rise: analysis of period and birth cohort effects on recent trends. Ann Oncol 2012;23:3155-62
17 Edgren $\mathrm{G}$, Adami H-O, Weiderpass E, et al. A global assessment of the oesophageal adenocarcinoma epidemic. Gut 2013;62:1406-14.

18 Arnold M, Laversanne M, Brown LM, et al. Predicting the future burden of esophageal cancer by histological subtype: international trends in incidence up to 2030. Am J Gastroenterol 2017;112:1247-55.

19 Derakhshan MH, Arnold M, Brewster DH, et al. Worldwide inverse association between gastric cancer and esophageal adenocarcinoma suggesting a common environmental factor exerting opposing effects. Am J Gastroenterol 2016;111:228-39.

20 Mitchell DR, Derakhshan MH, Wirz AA, et al. The gastric acid pocket is attenuated in H. pylori infected subjects. Gut 2017;66:1555-62.

21 Spechler SJ. Cardiac mucosa: the heart of the problem. Gut 2015;64:1673-4.

22 Mosavi-Jarrahi A, Mohagheghi MA. Epidemiology of esophageal cancer in the highrisk population of Iran. Asian Pac J Cancer Prev 2006;7:375-80.

23 Lin Y, Totsuka Y, He Y, et al. Epidemiology of esophageal cancer in Japan and China. J Epidemiol 2013;23:233-42.

24 Somdyala NI, Bradshaw D, Gelderblom WC, et al. Cancer incidence in a rural population of South Africa, 1998-2002. Int J Cancer 2010;127:2420-9.

25 Vizcaino AP, Parkin DM, Skinner ME. Risk factors associated with oesophageal cancer in Bulawayo, Zimbabwe. Br J Cancer 1995;72:769-73.

26 Ocama P, Kagimu MM, Odida M, et al. Factors associated with carcinoma of the oesophagus at Mulago Hospital, Uganda. Afr Health Sci 2008;8:80-4.

27 Pandeya N, Olsen CM, Whiteman DC. Sex differences in the proportion of esophageal squamous cell carcinoma cases attributable to tobacco smoking and alcohol consumption. Cancer Epidemiol 2013;37:579-84.

28 Brown LM, Hoover R, Silverman D, et al. Excess incidence of squamous cell esophageal cancer among US black men: role of social class and other risk factors. Am J Epidemiol 2001:153:114-22.

29 Sheikh M, Poustchi H, Pourshams A, et al. Individual and Combined Effects of Environmental Risk Factors for Esophageal Cancer Based on Results From the Golestan Cohort Study. Gastroenterology 2019;156:1416-27.

30 Middleton DR, Menya D, Kigen N, et al. Hot beverages and oesophageal cancer risk in Western Kenya: findings from the ESCCAPE case-control study. Int I Cancer 2019;144:2669-76.

31 Lao-Sirieix P, Fitzgerald RC. Screening for oesophageal cancer. Nat Rev Clin Oncol 2012;9:278.

32 Markar SR, Wiggins T, Antonowicz S, et al. Assessment of a noninvasive exhaled breath test for the diagnosis of oesophagogastric cancer. JAMA Oncol 2018;4:970-6.

33 Offman J, Muldrew B, O'Donovan M, et al. Barrett's oESophagus trial 3 (BEST3): study protocol for a randomised controlled trial comparing the Cytosponge-TFF3 test with usual care to facilitate the diagnosis of oesophageal pre-cancer in primary care patients with chronic acid reflux. BMC Cancer 2018;18:784.

34 Hoyo C, Cook MB, Kamangar F, et al. Body mass index in relation to oesophageal and oesophagogastric junction adenocarcinomas: a pooled analysis from the International beacon Consortium. Int J Epidemiol 2012;41:1706-18.

35 Kamangar F, Dawsey SM, Blaser MJ, et al. Opposing risks of gastric cardia and noncardia gastric adenocarcinomas associated with Helicobacter pylori seropositivity. J Natl Cancer Inst 2006:98:1445-52.

36 Arnold M, Park JY, Camargo MC, et al. Is gastric cancer becoming a rare disease? a global assessment of predicted incidence trends to 2035. Gut 2020;69:823-9.

37 Hooi JKY, Lai WY, Ng WK, et al. Global prevalence of Helicobacter pylori infection: systematic review and meta-analysis. Gastroenterology 2017;153:420-9.

38 Camargo MC, Anderson WF, King JB, et al. Divergent trends for gastric cancer incidence by anatomical subsite in US adults. Gut 2011;60:1644-9.

39 Diet, Nutrition, Physical activity, and Stomach Cancer. Continuous update project report. world cancer research fund / American Institute for cancer research 2016.

40 Lee KS, Oh DK, Han MA, et al. Gastric cancer screening in Korea: report on the National cancer screening program in 2008. Cancer Res Treat 2011;43:83-8.

41 Nakamura Y, Takeshita M, Hirota Y, et al. An evaluation of gastric cancer screening program in Hisayama, Japan. Gastroenterol Jpn 1977;12:427-34.

42 Hamashima C. Guideline development group for gastric cancer screening G. update version of the Japanese guidelines for gastric cancer screening. Jpn J Clin Oncol 2018:48:673-83

43 Jun JK, Choi KS, Lee H-Y, et al. Effectiveness of the Korean National cancer screening program in reducing gastric cancer mortality. Gastroenterology 2017;152:1319-28.

44 Ford AC, Forman D, Hunt RH, et al. Helicobacter pylori eradication therapy to prevent gastric cancer in healthy asymptomatic infected individuals: systematic review and meta-analysis of randomised controlled trials. BMJ 2014;348:93174.

45 Areia M, Carvalho R, Cadime AT, et al. Screening for gastric cancer and surveillance of premalignant lesions: a systematic review of cost-effectiveness studies. Helicobacter 2013;18:325-37.

46 Lindblad M, Ye W, Lindgren A, et al. Disparities in the classification of esophageal and cardia adenocarcinomas and their influence on reported incidence rates. Ann Surg 2006;243:479-85.

$47 \mathrm{McColl} \mathrm{KEL}$, Going JJ. Aetiology and classification of adenocarcinoma of the gastrooesophageal junction/cardia. Gut 2010;59:282-4. 